\title{
Genome-wide association study revealed genetic variations of $A B A$ sensitivity controlled by multiple stress-related genes in rice
}

Lei Peng ${ }^{\dagger}$, Tingting Xie ${ }^{* \dagger}$, Zilong Guo, Xiaokai Li, Yu Chang, Haifu Tu, Shengchang Wang, Nai Wu, Yilong Yao and Lizhong Xiong ${ }^{*}$ (iD

\begin{abstract}
Abscisic acid (ABA) is a critical phytohormone that regulates multiple physiological processes including plant growth and stress tolerance. The core ABA signaling pathway has been well established, but genetic variations mediating $A B A$ responses remain largely unknown. In this study, we performed genome-wide association study (GWAS) to identify loci and genes associated with ABA sensitivity (reflected by seed germination inhibition by ABA) in a panel of 425 rice accessions. The seed germination assay revealed that Aus and indica rice had stronger ABA sensitivity than japonica rice. A total of 48 non-redundant association loci were detected in the indica subpopulation and whole population, and 386 genes in these loci were responsive to ABA or abiotic stresses. Eight association loci were overlapped with previously reported loci for yield under drought stress or for droughtindicative image traits. Haplotype analyses of important candidate genes such as OsSAPK6, a key component in the ABA signaling core, were performed to identify key SNPs/InDels that may affect gene functions through promoter activity regulation, amino acid variation, or gene splicing. These results provide insights into the genetic basis of ABA sensitivity related to stress responses.
\end{abstract}

Keywords: ABA sensitivity, Seed germination, Stress response, Rice

\section{Introduction}

Abscisic acid (ABA) is an important stress hormone for the responses and adaptation of plants to abiotic stresses by controlling multiple processes of plant growth and development. ABA plays a crucial role in various physiological processes, such as stomatal closure, cuticular wax accumulation, leaf senescence, bud dormancy, growth inhibition, osmotic regulation, and seed germination (reviewed in (Chen et al. 2020)). Due to the discovery of ABA receptors, our understanding of the ABA signaling pathway has been largely deepened (Ma et al. 2009; Park et al. 2009). ABA signaling core,

\footnotetext{
* Correspondence: tingtingxie@mail.hzau.edu.cn; lizhongx@mail.hzau.edu.cn ${ }^{\dagger}$ Lei Peng and Tingting Xie contributed equally to this work.

National Key Laboratory of Crop Genetic Improvement, Hubei Hongshan Laboratory, Huazhong Agricultural University, Wuhan 430070, China
}

including ABA receptor PYRABACTIN RESISTANCE/PYRABACTIN RESISTANCE-LIKE/REGULATORY COMPONENTS OF THE ABSCISIC ACID RECEPTOR (PYR/PYL/ RCAR), clade-A type- $2 \mathrm{C}$ protein phosphatase (PP2C), and SNF1-related protein kinase 2 (SnRK2), have been wellknown for their canonical molecular mechanisms in multiple ABA-induced plant physiological processes (Chen et al. 2020; Cutler et al. 2010). When there is no ABA, PP2Cs dephosphorylate SnRK2 kinases and inhibit their activities (Umezawa et al. 2009; Vlad et al. 2009). When ABA is bound by its receptors PYR/PYL/RCAR, PP2Cs are inhibited by the receptors through direct binding, leading to activation of SnRK2 (Ma et al. 2009; Park et al. 2009; Tischer et al. 2017). Activated SnRK2s phosphorylate substrate proteins such as transcription factors including ABSCISIC 
ACID INSENSITIVE 5 (ABI5) which subsequently regulate arrays of genes related to stress responses (Fujii and Zhu 2009).

Diverse proteins are involved in ABA signaling by regulating activity of the $\mathrm{ABA}$ signaling core components. ABA receptor PYL4 can be phosphorylated and inhibited by TARGET OF RAPAMYCIN (TOR) kinase, which is in turn inhibited by ABA through SnRK2-mediated protein phosphorylation of the REGULATORY-ASSOCIATED PROTEIN OF TOR 1B (RAPTOR1B) (Wang et al. 2018a). By contrast, PYR/PYL/RCAR members have been shown to be phosphorylated and activated by receptor-like cytoplasmic kinase CARK1 (Li et al. 2019; Zhang et al. 2018). According to a recent study, SnRK2.6 reactivation requires another essential signaling component belonging to B3 subfamily of Raf-like MAP kinase kinase kinase (MAPK KK) family (Takahashi et al. 2020). PP2C activity can be stabilized (Li and Liu 2012) or increased (Yu et al. 2012) by small GTP-binding protein Rho-like GTPase protein (ROP) family, which is a negative regulator of ABA signaling. ENHANCER OF ABA CO-RECEPTOR 1 (EAR1), another negative regulator of $\mathrm{ABA}$, can enhance $\mathrm{PP} 2 \mathrm{C}$ activities through direct interaction with $\mathrm{N}$-terminal autoinhibitory domain of PP2Cs (Wang et al. 2018b). In addition, several proteins including two U-Box E3 ligases (PUB12 and PUB13), two Ring-type E3 ligases (RGLG1 and RGLG5), and two adaptor proteins (DET1-AND DDB1-ASSOCIATED 1 [DDA1] and BTB/POZ AND MATH DOMAIN protein $[\mathrm{BPM}]$ ) can regulate ABA signaling by degrading PYR/PYL/RCAR receptors or PP2Cs (Julian et al. 2019; Garcia-Leon et al. 2019; Wu et al. 2016; Kong et al. 2015; Irigoyen et al. 2014).

The timing of seed germination is crucial for plant growth in correct seasons. ABA inhibits germination but promotes seed maturation and dormancy (Koornneef et al. 2002). In 1990s, screening mutants of seed germination inhibition was an important approach for studying ABA signaling (Allen et al. 1999; Finkelstein and Lynch 2000; Giraudat et al. 1992; Huijser et al. 2000; Leung et al. 1994; Leung et al. 1997; Rodriguez et al. 1998). The seed germination-inhibited mutants were named $\mathrm{ABI}$ (ABA insensitive) mutants. $A B I 1$ and $A B I 2$, encoding two PP2C protein phosphatases in the ABA signaling core, play essential roles in the regulation of seed germination (Leung et al. 1994; Leung et al. 1997). ABI3, $A B I 4$, and $A B I 5$, encoding transcription factors in the ABA signaling pathway, are also important to seed dormancy (Finkelstein and Lynch 2000; Giraudat et al. 1992; Huijser et al. 2000; Finkelstein et al. 1998). Besides the PYR/PYL/RCAR-PP2C-SnRK2 signaling core, an increasing number of proteins have been identified with roles in the complicated ABA molecular network for seed germination. These proteins include LEAFY COTYLEDON2 (LEC2), FUSCA3 (FUS3) (structurally related to $\mathrm{ABI}$ /VP1), calcium-dependent protein kinases (CPKs), SnRK2-interacting calcium sensor (SCS), calcineurin B-like protein 9 (CBL9) and CBL-interacting protein kinase 3 (CIPK3), and late embryogenesis abundant (LEA) class proteins (reviewed in (Nakashima and Yamaguchi-Shinozaki 2013)).

Although the ABA signaling core have been intensively studied and widely accepted, the ABA signaling network and genetic variation of $A B A$ response need to be further revealed, especially in economically important crops. In this study, genome-wide association study (GWAS) was used to identify genetic loci of ABA sensitivity, indicated by seed germination inhibition by $\mathrm{ABA}$, in a global collection of 425 rice accessions. New association loci for ABA sensitivity and novel candidate genes involved in ABA signaling and/or stress tolerance were identified. Our results provided an overall scenario on the natural variation of $\mathrm{ABA}$ sensitivity in rice germplasms and identified numbers of significantly associated loci and candidate genes involved in the ABA signaling and multiple stress responses.

\section{Results \\ Population structure and phenotypic variation in $A B A$ sensitivity in rice germplasms}

Considering that the population size in this study (425 accessions) was smaller than the original population (529 accessions) used for GWAS (Chen et al. 2014), a principal component (PC) analysis based on 4.3 million SNPs was conducted to examine the population structure. The results showed that indica (263 accessions), japonica (104 accessions), and Aus (42 accessions) subpopulations were separated by PC1 (explaining 63.28\% of the total genetic variation) and $\mathrm{PC} 2$ (explaining $20.00 \%$ of the total genetic variation) (Fig. 1a). This population structure was essentially the same as that of the original population.

Seed germination inhibited by ABA has been commonly used as criteria to evaluate ABA sensitivity. To investigate the genetic variation of $A B A$ sensitivity in the rice population, matured and dried seeds were used for germination with or without ABA treatment. Seed germination percentage under non-treatment conditions (CKG) and ABA-treated conditions (ABAG) as well as relative germination percentage (REG), ratio of ABAG/ CKG, were investigated in the whole population containing all 425 rice accessions, and the three major subpopulations. Some representative images of ABA inhibited seed germination phenotypes and corresponding REG are shown in Fig. 1b. CKG in indica, japonica, Aus, and the whole population ranged in $29.51 \%-99.44 \%$, $29.98 \%-100 \%, \quad 42.25 \%-99.46 \%$, and $29.51 \%-100 \%$, respectively, thus indicating that large variations in seed 


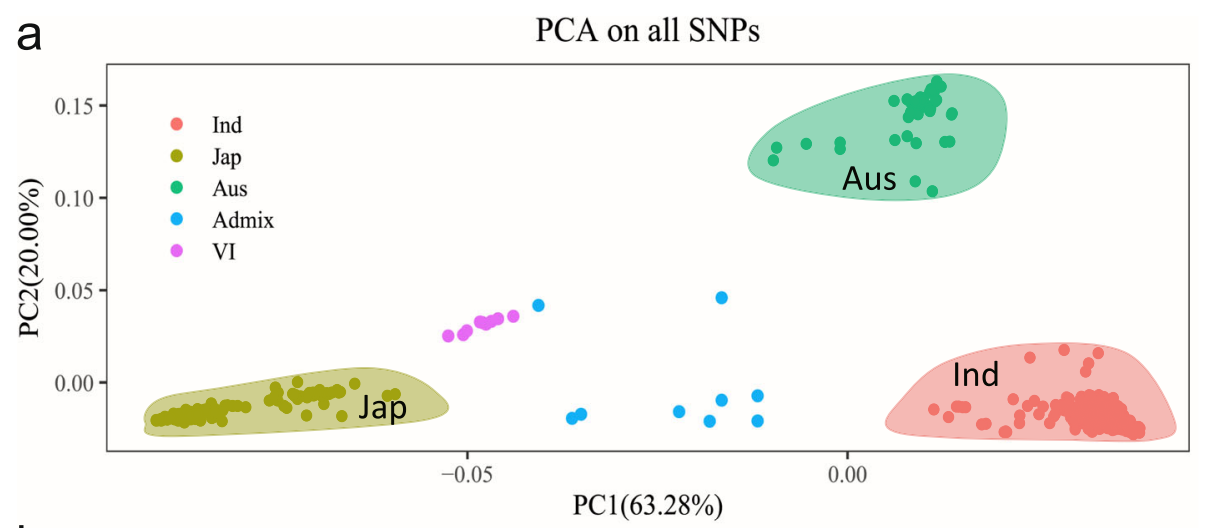

b

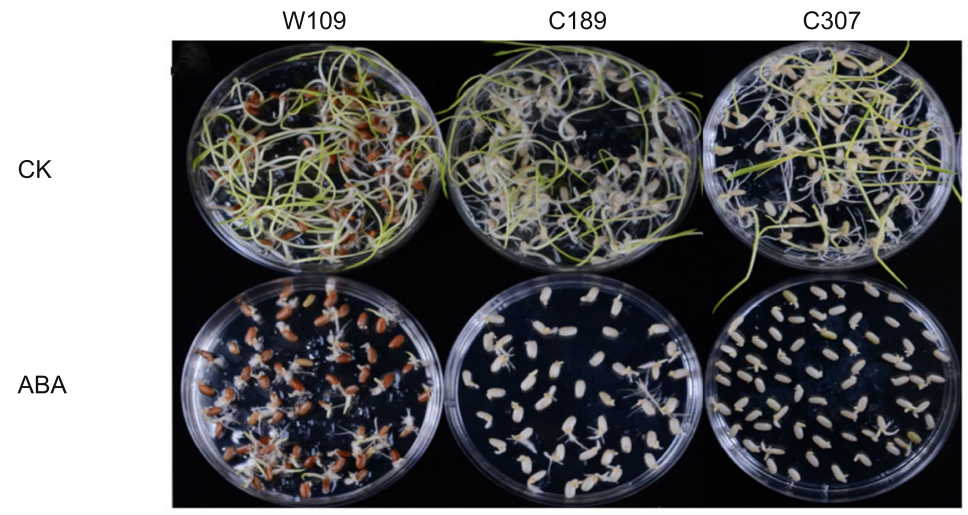

C

$91.81 \%$

$45.87 \%$

$12.70 \%$

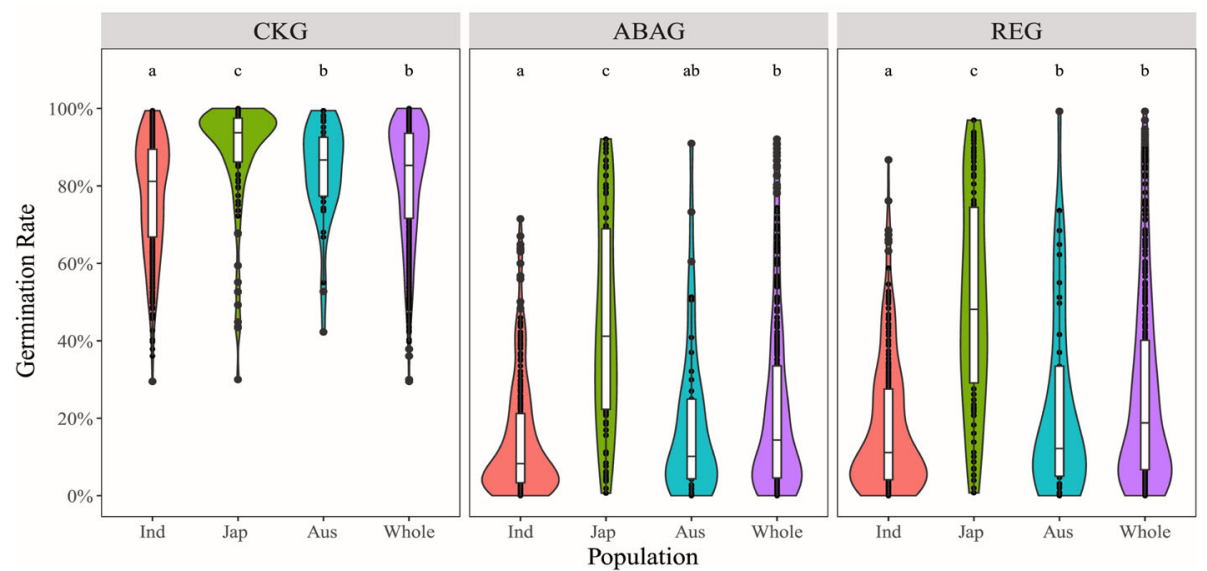

Fig. 1 Population structure analysis and seed germination rate variation within the subpopulations and whole population. a Principal component (PC) analysis for all 425 accessions, including indica (Ind), japonica (Jap), Aus (Aus), and some intermediate (VI and Admix). b Some representative images of ABA inhibited seed germination phenotypes and corresponding REG. $\mathbf{c}$ Variation and statistical analysis for seed germination rate of CK group (CKG) and ABA-treated group (ABAG), and the relative germination rate (REG, $A B A G / C K G \times 100 \%)$ in the subpopulations and whole population

germination existed in rice germplasms (Fig. 1c). ABAG in indica, japonica, Aus, and the whole population varied in the range of $0.00 \%-71.48 \%, 0.06 \%-92.13 \%, 0 \%-$ $90.98 \%$, and $0 \%-92.13 \%$, respectively. The mean seed germination rate under $\mathrm{ABA}$ treatment was significantly lower than that in the non-treatment control $(P<0.001)$, suggesting that the inhibition of seed germination by
ABA was effective and reliable in this experiment. The range of REG variations in indica, japonica, Aus, and the whole population were $0.00 \%-86.70 \%, 0.07 \%-96.98 \%$, $0.00 \%-99.26 \%$, and $0.00 \%-99.26 \%$, respectively. These results clearly indicated there was a large genetic variation in ABA sensitivity in the subpopulations and the whole population. Among the whole populations, 11 
indica accessions (C019, C020, C039, C069, C147, C159, C168, W058, W180, W187, W259) and 3 Aus accessions (W108, W131, W292) showed very strong hypersensitivity to ABA (REG $<0.50 \%$ ), while 7 japonica accessions (C085, C111, C146, W053, W060, W109, W193), 1 Aus accession (W330) and 1 Admix accession (W097) showed no obvious sensitivity to ABA (REG > 90.00\%) (Supplementary Table 1). Statistical analysis revealed significant difference in the seed germination ability among the subpopulations. The mean of CKG (88.52\%), ABAG (45.77\%) and REG (50.10\%) in japonica were significantly higher than those in indica $(77.26 \%, 14.52 \%$, $17.77 \%$, respectively) and $A u s(83.47 \%, 18.98 \%, 22.94 \%$, respectively) $(P<0.05$, Fig. 1b). This result indicates that japonica varieties are generally weaker in seed dormancy and less sensitive to ABA than indica and Aus varieties.

\section{Association mapping of ABAG and REG}

Since the seed germination ability varied under normal germination conditions, REG under ABA treatment was also used for association mapping of ABA sensitivity in addition to ABAG. Factored Spectrally Transformed Linear Mixed Model (FaST-LMM) was used to identify association signals in the indica, japonica and whole populations, using a total of 4.3 million SNPs in the 425 rice accessions. Several significant association signals were detected in the whole population and the indica subpopulation, as indicated by Manhattan and Quantilequantile plots (Fig. 2). However, no significant signal was detected in the japonica subpopulation. Although marginally significant SNPs were detected for ABAG or REG in the Aus subpopulation, they were not subjected for further analyses because of small population size.

A total of 131 and 188 SNPs for ABAG and 100 and 67 SNPs for REG were detected by GWAS in the indica subpopulation and the whole population, respectively, at a significance level of $-\log (P)>6$. Based on reported genome-wide linkage disequilibrium (LD) decay distance (Huang et al. 2010; Huang et al. 2012), all variants in high LD $\left(r^{2}>0.6\right)$ of significant SNPs within a range of $150 \mathrm{~kb}$ were merged as an association locus. Adjacent association loci were merged into single association locus when they were overlapped. The association loci with a length of shorter than $5 \mathrm{~kb}$ were ignored.

In the indica subpopulation, 17 and 12 association loci were detected for ABAG and REG, respectively. In the whole population, 22 and 18 association loci were detected for ABAG and REG, respectively. A total of 48 non-redundant loci were detected for ABAG or REG in, at least, one population (Supplementary Table 2). These results suggest that genetic variations of ABA sensitivity may be controlled by multiple loci in rice.

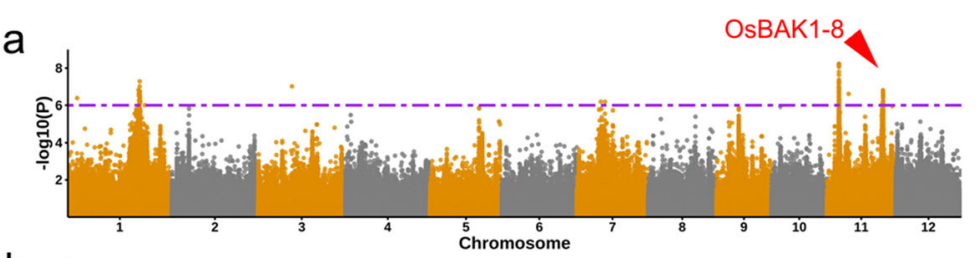

b

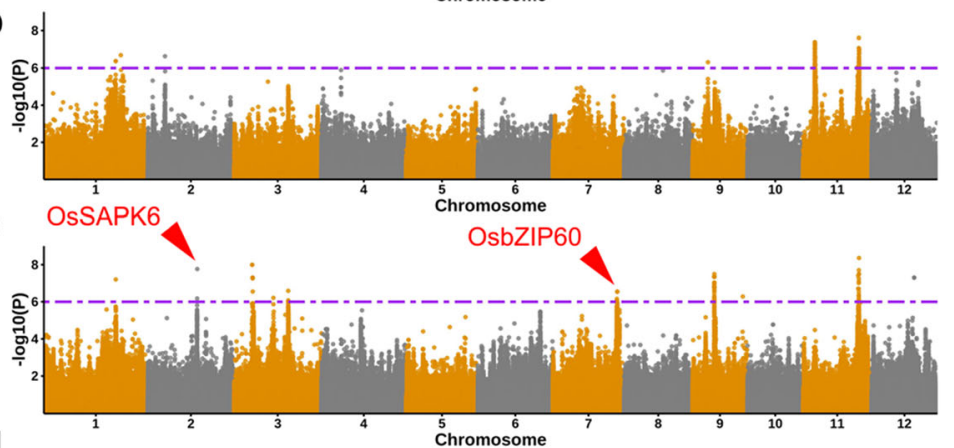

d
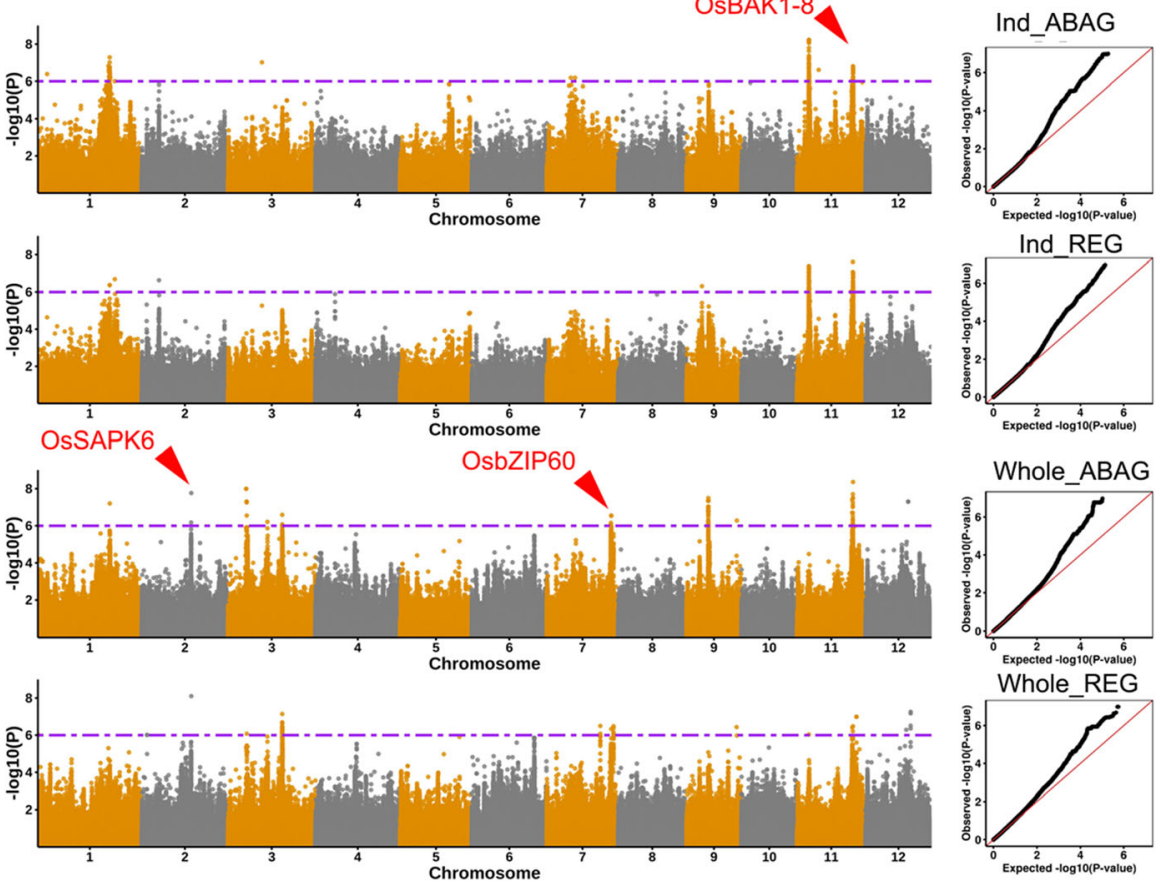

C

Whole ABAG
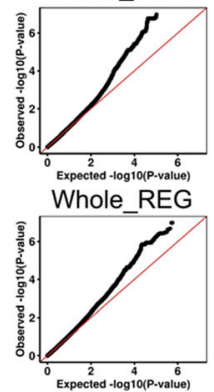

Fig. 2 GWAS for seed germination rate in the indica subpopulation and whole population. Manhattan and Quantile-quantile plots are shown for ABAG in the indica (a), REG in the indica (b), ABAG in the whole populations (c), and REG in the whole population (d) using FaST-LMM. Red arrows indicate the genes are considered to be important candidates related to ABA sensitivity. Dashed horizontal line for each population indicates the suggestive threshold $\left(P=1.0 \times 10^{-6}\right)$ 


\section{Genes related to $A B A$ or stress responses within the} significant loci of REG and ABAG

In order to identify genes involved in ABA sensitivity, all the non-transposon genes in the association loci of REG and ABAG were extracted from the annotated Nipponbare reference genome. A total of 590 non-redundant and non-transposon genes were annotated in the 48 nonredundant association loci distributed in chromosome 1, 2, 3, 7, 9, 11, and 12 (Fig. 3a, Supplementary Table 3). There were 329 and 135 genes in ABAG and REG association loci, respectively, in the indica subpopulation, and 191 and 206 genes in ABAG and REG association loci, respectively, in the whole population (Fig. $3 \mathrm{~b}$ ). In total, 221 genes were identified in the common loci for ABAG and REG. Among them, 4 genes including LOC_ Os11g39330, LOC_Os11g39350, LOC_Os11g39360, and LOC_Os11g39370 (OsBAK1-8) were presented in the loci for ABAG and REG in both the indica subpopulation and the whole population.

By searching the expression levels of 590 genes in a public database (https://tenor.dna.affrc.go.jp), a total of 386 non-redundant genes were found to be responsive to ABA or stresses. Among them, 327, 282, and 293 genes were responsive to $\mathrm{ABA}$, drought, and osmotic stress, respectively, and 219 genes were responsive to all the three treatments (Fig. 3c). These results suggest that $\mathrm{ABA}$ and stress-responsive genes are highly enriched in the loci for ABA sensitivity.

According to the function annotation and/or reported functions of the genes in the significantly associated loci for ABAG and REG, at least nine genes, including OsSAPK6 (LOC_Os02g34600) (Saha et al. 2014), bZIP60 (LOC_Os07g44950) (Xu et al. 2011), OsBAK1-8 (LOC_ Os11g39370) (Khew et al. 2015), OsCIPK30 (LOC_ Os01g55440) (Kanwar et al. 2014), OsCIPK12 (LOC_ Os01g55450) (Kanwar et al. 2014), OsCBL10 (LOC_
Os01g51420) (Kanwar et al. 2014), OsFUS3 (LOC_ Os01g51610) (Brocard-Gifford et al. 2003), and OsBPM (LOC_Os07g20130) (Julian et al. 2019), were directly or indirectly related to ABA signaling and its regulation.

\section{Haplotype analysis of selected candidate genes}

To further test which of the ABA signaling-related candidate genes in the association loci were involved in ABA sensitivity, all SNPs or InDels in the candidate genes were extracted from RiceVarMap 2.0 (Zhao et al. 2015) for haplotype analysis. All nonsynonymous SNPs/InDels within the coding regions and the most significantly associated SNPs/InDels in promoter regions were selected for haplotype analysis. Among the ABA and stress-responsive genes in the loci associated with REG or ABAG, haplotype analysis results of three candidate genes including OsSAPK6, bZIP60, and OSBAK1-8 were presented in Fig. 4 and described as follows. The information of key SNPs and InDels in each candidate gene was shown in Supplementary Table 4.

OsSAPK6 is a member of SnRK2 protein kinase family in the ABA signaling core (Saha et al. 2014). This gene is responsive to drought, osmotic stress, and ABA treatment (Supplemental Fig. 1). Seven SNPs in OsSAPK6 were considered as possible causal variations between haplotype 1 (hap1) and haplotype 2 (hap2) (Fig. 4a). Two SNPs (vg0220751393 and vg0220751504) were located in two putative AP2/ERF-binding elements. Two SNPs (vg0220751393 and vg0220752702) were located in putative NF-YA/YB/YC elements. Vg0220751504, vg0220753260 and vg0220753096 were located in a putative MYB-binding element, a putative bZIP-binding element, and a putative ABA-responsive element (ABRE), respectively. REG was significantly different between hap1 (15.5\%, 303 accessions) and

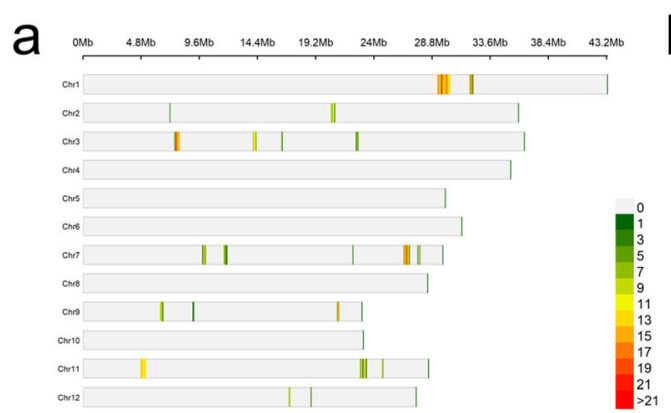

Distribution of 590 genes within $0.1 \mathrm{Mb}$ window size

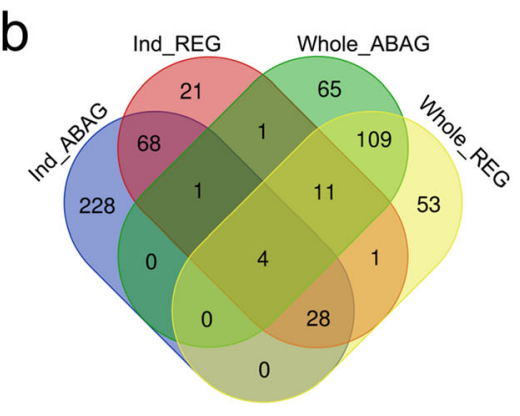

Candidate genes (590)

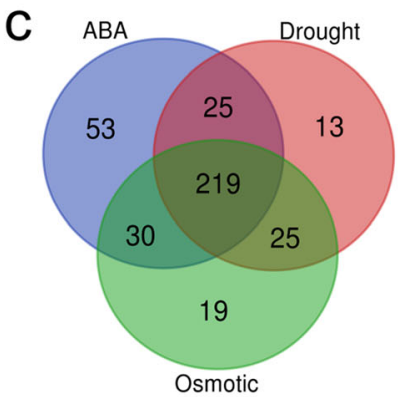

Stress/ABA-response genes (384)

Fig. 3 Gene analysis within the association loci. a. Chromosomal distribution of 590 genes in the loci associated with ABA sensitivity. Color scale indicates the number of genes in the associated loci. b. Venn diagram showing the distribution of the genes in the associated loci for ABAG in the indica subpopulations (Ind_ABAG) and whole population (Whole_ABAG), and the genes in the associated loci for REG in the indica subpopulations (Ind_REG) and whole population (Whole_REG). c. Venn diagram showing the number of the genes from the association loci are responsive (threshold of 2-fold-change) to ABA, drought, and/or osmotic stresses. The relative expression levels of these genes in the treatment of $\mathrm{ABA}$ and drought or osmotic stress were extracted from a public database (https://tenor.dna.affrc.go.jp) 


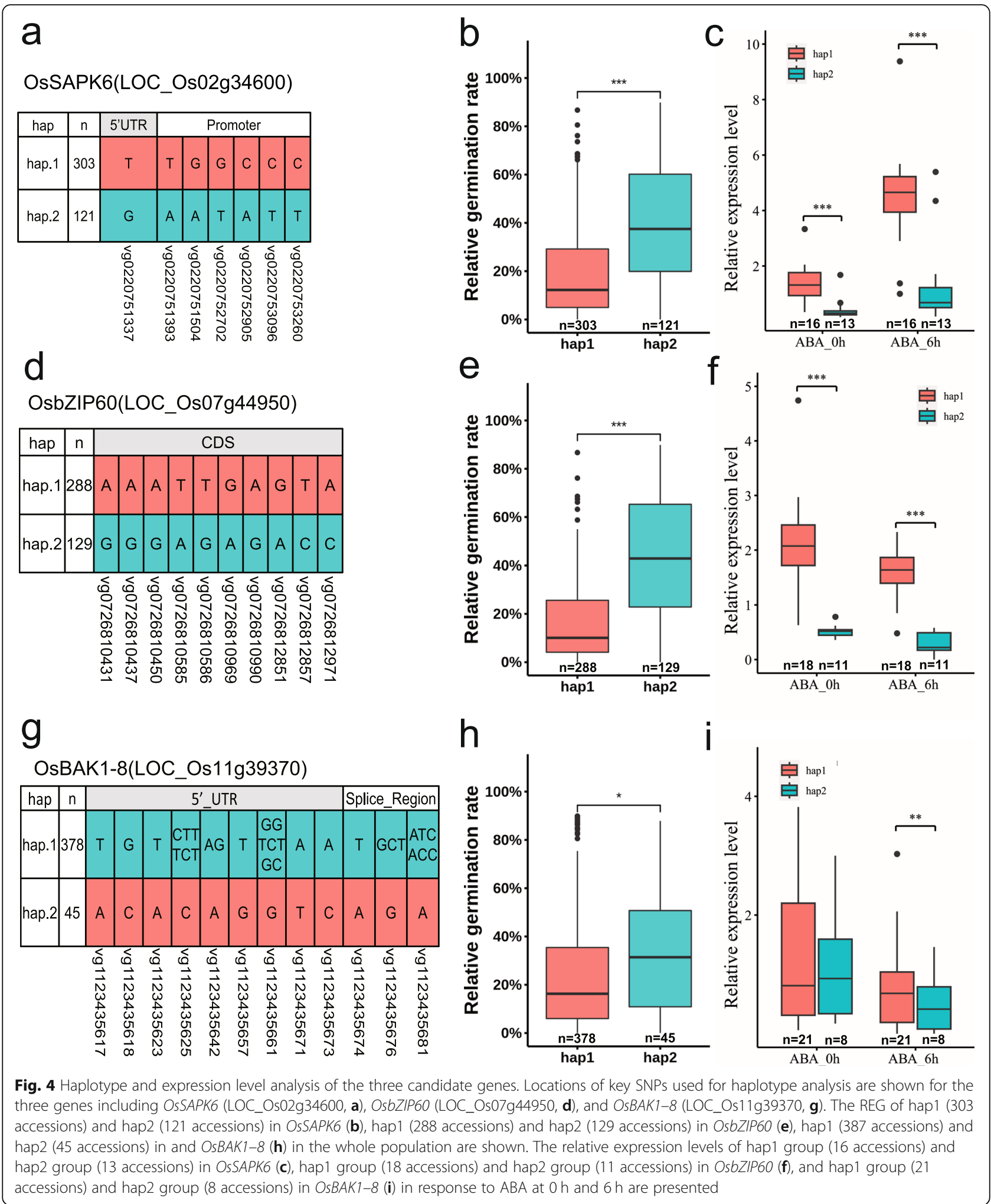

hap2 $(30.5 \%, 121$ accessions) in the whole population $(P<0.001$, Fig. 4b). These results indicate that the expression of OsSAPK6 might be affected by these SNPs. We performed qPCR to detect the expression levels of OsSAPK6 in seedling leaves with or without ABA treatment in randomly selected ABA-insensitive and ABA-sensitive rice accessions. These accessions were divided into two groups to compare the 
expression levels of OsSAPK6. The hap1 group (16 accessions) had significantly higher expression levels of OsSAPK6 than hap2 group (13 accessions) under both normal and ABA treatment conditions, and the difference under ABA treatment was more obvious (Fig. 4c). This result indicates that the difference in the expression levels of OsSAPK6 between the two haplotypes might contribute to the difference in $\mathrm{ABA}$ sensitivity.

OsbZIP60 was up-regulated by heat and drought stresses as well as ABA treatment (Supplemental Fig. 1). Ten nonsynonymous SNPs in the coding region of OsbZIP60 caused amino acid changes including Gly172Ser, Ala174Thr, Gly178Asp, Gln223Leu, Gln223His, Glu351Gly, Gly358Glu, Gly484Asp, Thr486Ile, and Ala524Glu between two haplotypes (Fig. 4d). Among them, Gly172Ser occurred in bZIP domain, and it may affect the function of OsbZIP60. There was also a significant difference in REG between the two haplotypes of OsbZIP60 in the whole population $(P<0.001$, Fig. 4e). Although there were no significant variations detected in the OsbZIP60 promoter, the expression levels showed a significant difference between the two haplotypes (16 accessions of hap1 and 11 accessions of hap2) (Fig. 4f). These results suggest that, in addition to the coding variations of OsbZIP60, other distal regulatory elements may be involved in the function of this gene in the regulation of ABA sensitivity.

OsBAK1-8 encodes a homologue of Arabidopsis BAK1, which is responsive to ABA and can interact with SnRK2.6 (Khew et al. 2015; Shang et al. 2016). OsBAK18 is slightly responsive to drought stress and ABA treatment (Supplemental Fig. 1). The two major haplotypes were identified for OsBAK1-8 with seven SNPs and five InDels (Fig. 4g). There was also a significant difference in REG between the two haplotypes in the whole population $(P<0.05$, Fig. 4h). An important InDel (vg1123435674) located at the splice acceptor sequence of GT-AG can generate different open reading frames, which may affect the function OsBAK1-8 related to ABA sensitivity. It cannot be excluded that the SNPs in 5'UTR may affect the expression level of OsBAK1-8. However, qPCR analysis showed that, unlike the former two candidate genes, there was no difference in the expression levels of OsBAK1-8 between the two haplotypes (21 accessions of hap1 and 8 accessions of hap2) (Fig. 4i). This result suggests that further studies may focus on the variation at splicing site of OsBAK1-8 to reveal its relationship with $\mathrm{ABA}$ sensitivity in rice.

\section{Discussion}

Although the ABA signaling core has been intensively studied and widely accepted, the genetic variation of ABA signaling and its physiological "output" (collectively called ABA sensitivity) remain largely unknown.
Therefore, identification of more genetic components of ABA sensitivity is critical to illustrate how ABA signaling fine tunes the plant responses to environmental cues and stresses. In this study, we investigated the genetic variation of $A B A$ sensitivity (in terms of the seed germination capability under $\mathrm{ABA}$ treatment) for the first time in a large rice panel containing 425 accessions worldwide. We observed that the seed germination capability without ABA treatment dramatically varied in the indica subpopulation, but less variation was observed in the japonica subpopulation. Similar result has been reported in an early study on rice seed dormancy (Magwa et al. 2016). Our results also showed that the indica or Aus subpopulation were generally more sensitive to ABA than the japonica subpopulation. In accordance with the variation difference in ABA sensitivity, GWAS detected 17 association loci for ABAG and 12 association loci for REG in the indica subpopulation, but failed to detect significant association loci in the japonica subspecies. However, it cannot be excluded that minor effective QTLs may be detected in the japonica subspecies if lower concentration of $\mathrm{ABA}$ is used in the treatment.

Within the 48 non-redundant association loci (an average size of $98 \mathrm{~kb}$ ) detected by GWAS of ABAG and REG (Supplementary Table 2), 590 non-transposon genes were further analyzed for their candidacy related to ABA signaling and/or metabolism (Supplementary Table 3). Surprisingly, very few genes in these loci matched the expected functions in the ABA signaling or metabolism pathways. The PYL-PP2C-SnRK2-ABREB (ABI5/bZIP) module has been well recognized in the core ABA signaling pathway. The rice genome contains 12 OsPYLs (He et al. 2014; Kim et al. 2012), 10 clade A PP2Cs, 10 SnRK2 protein kinases family (SAPKs), and 11 bZIP transcription factors in subgroup A with functions similar to ABI5 (Zong et al. 2016). It was expected that some of these genes in the ABA signaling core might have significant variations associated with $A B A$ sensitivity. However, according to our results, only OsSAPK6 was detected with an associations with ABAG and REG in the whole population. One possibility could be that the functions of different members in the core ABA signaling gene families have been differentiated in the ancestral species of cultivated rice to fine tune not only ABA signaling but also other processes in plant growth and adaptation to various environmental cues, and therefore most of these core genes have no or marginal natural variations in $\mathrm{ABA}$ sensitivity in terms of the seed germination inhibition by ABA. In fact, only a few important natural variations have been reported for the core genes of other phytohormone signaling pathways, and the variations controlling many important agronomic traits occurred mainly in the regulatory or downstream genes of core signaling pathways. Another 
possibility could be that most of the germplasms or varieties used in this study are lowland or irrigated rice that have been domesticated or selected under non-stressed conditions, and therefore the core ABA signaling genes related to stress tolerance may be not necessary to be selected in lowland rice, unlike other upland crops such as maize (Shikha et al. 2017; Xiang et al. 2017).

OsSAPK6, a component in the ABA signaling core, has been found to be associated with ABA sensitivity. Previous studies have reported that OsSAPK6 is ABAinducible and it is related to osmotic stress signaling (Chae et al. 2007; Kobayashi et al. 2004, 2005). OsSAPK6 can interact with and phosphorylate OsbZIP10 in response to ABA signaling in rice (Chae et al. 2007). OsSAPK6, as well as OsSAPK2 and OsSAPK9, can also phosphorylate and activate OsbZIP46 to pass on ABA signaling to gene expression (Kim et al. 2015; Tang et al. 2012). Our results showed that most of the significantly associated SNPs in OsSAPK6 were located in its promoter or 5'UTR region, and the expression levels of OsSAPK6 were higher in hap1 than that in hap2, indicating that the expression levels of OsSAPK6 might vary in rice germplasms, thereby affecting $\mathrm{ABA}$ sensitivity. In fact, the two haplotypes of OsSAPK6 based on the significant SNPs in the promoter showed significant difference in both ABA sensitivity and the expression level of the gene itself. Our previous study showed that overexpression of OSSAPK6 enhanced ABA sensitivity as well as drought and heat tolerance (Chang et al. 2017), which also supported the important role of transcriptional regulation of OsSAPK6 in ABA signaling. The significant variations in the promoter may be targets for further identification of transcription factors regulating OsSPAK6 expression or for designing molecular marker of superior allele for breeding selection.

In addition to OsSAPK6, at least eight genes with putative functions in regulating or amplifying ABA signaling were detected with significant associations with ABA sensitivity. LOC_Os01g51610 is a FUS3 homologue closely related to $\mathrm{ABI} 3 / \mathrm{VP} 1$ which regulates seed germination by interacting with $\mathrm{ABI} 4$ and $\mathrm{ABI} 5$ (BrocardGifford et al. 2003). LOC_Os07g20130 is annotated as a homolog of BPM which regulates seed germination by affecting degradation of PP2Cs (Julian et al. 2019), and it was reported to be up-regulated by heat and drought stresses (Xu et al. 2011) as well as ABA treatment (https://tenor.dna.affrc.go.jp). The haplotype analysis suggested that the variations in the coding region of OsbZIP60 may mainly contribute to the function of this gene in ABA response. Nevertheless, other distal regulatory elements may be also involved in the regulation of OsbZIP60 since the two haplotypes showed difference in the expression levels of this gene under ABA treatment. OsBAK1-8, a homologue of BAK1, might regulate seed germination by affecting the activity of SnRK2 (Khew et al. 2015). It was reported that BAK1 can form a complex with OST1 (SnRK2.6 or SRK2E) near the plasma membrane and that ABA can enhance the formation of the complex in Arabidopsis (Shang et al. 2016). The haplotype analysis and qPCR analysis suggested that the variation at a splicing site of OsBAK1-8 might be an important clue to track down the causal variation of this gene for its contribution to ABA sensitivity. The haplotype and expression level analyses of these genes provided additional evidence for their roles in $\mathrm{ABA}$ sensitivity, and further genetic and molecular studies of these candidate genes will uncover their exact functions related to ABA signaling or regulation. It should be noted that there are some reported cases in which the functional variants are large structure variations (Yuan et al. 2021), however the structure variations cannot be detected in the dataset used in this study. Large structure variations can be further excluded or confirmed by re-sequencing the genomic sequences covering the coding and regulatory regions of the important candidate genes in a condensed panel of the population or by waiting for the update of RiceVarMap with large structure variations included in the future.

It is interesting to notice that three calcium signalingrelated genes, including OsCIPK30, OsCIPK12 and OsCBL10, were detected with significant associations with ABA sensitivity. In Arabidopsis, several CIPKs and CBLs have been reported to regulate ABA signaling. For example, CIPK11 phosphorylated ABI5 and mutation of the phosphorylation site in $A B I 5$ resulted in higher trans-activation activity of ABI5 (Zhou et al. 2015). CBL2 might be essential for appropriate ABA responses because seed germination in the $c b l 2$ mutant was hypersensitive to ABA (Batistic et al. 2012). CBL9 and CIPK3 can interact with each other to regulate the ABA responses in seed germination (Pandey et al. 2008). It will be intriguing to reveal whether and how OsCIPK30, OsCIPK12 and OsCBL10 regulate ABA responses in rice.

ABA plays a crucial role in plant adaptation to adverse environmental stresses. In our previous study, drought resistance association loci were detected by GWAS in rice using a large population containing the accessions used the current study (Guo et al. 2018). Of these loci, seven lead SNPs were located in ABA sensitivity loci in this study. One of them was located in the same ABA sensitivity locus containing OSSAPK6 (Supplementary Table 2). We also found that two ABA sensitivity loci were overlapped with yield QTLs under drought stress conditions (Bhandari et al. 2020) (Supplementary Table 2). One of the loci also contained OsSAPK6 gene. However, no significant correlation was found between ABA sensitivity and drought resistance in the population used in this study (data not shown), suggesting that 
ABA sensitivity in terms of seed germination inhibition by ABA cannot completely reflect drought resistance because the latter is more complex and involves multiple processes at different growth and developmental stages. Nevertheless, the co-localization of several ABA sensitivity loci with drought resistance QTLs, such as the locus containing OsSAPK6, suggests that ABA sensitivity might be closely related to drought resistance. In addition, the expression data from TENOR database (https://tenor.dna.affrc.go.jp) showed that among 590 non-redundant genes in the ABA sensitivity loci, 327 (55.42\%), 282 (47.80\%), and 293 (49.66\%) genes were responsive to ABA, drought stress, and osmotic stress, respectively, and among these stress- or ABA-responsive genes, $57.03 \%$ (219 genes) were responsive to all the three treatments. Taken together, the partial overlapping of genetic loci for ABA sensitivity and drought resistance and the largely enriched stress-responsive genes in the $A B A$ sensitivity loci suggest that drought resistance in rice is closely related to $\mathrm{ABA}$ response but distinct $\mathrm{ABA}$ independent mechanism may also exist, which has been well recognized in Arabidopsis (Soma et al. 2021).

\section{Conclusions}

Genetic control of ABA sensitivity at seed germination was analyzed by genome-wide association mapping based on high density SNPs using 425 rice accessions. Totally, 48 non-redundant association loci were detected by GWAS of ABAG and REG in the indica or whole population. Eight loci of ABA sensitivity were overlapped with reported QTLs of drought resistance, one of which contained OsSAPK6, a component in ABA signaling core. This study provided new insights into the genetic basis of ABA sensitivity related to stress responses in rice, and the candidate genes identified in this study may be further explored for the genetic improvement of stress resistance.

\section{Methods}

\section{Plant materials}

An association population containing 529 rice accessions collected worldwide (Chen et al. 2014) were grown in the experimental station of Huazhong Agricultural University at Wuhan in 2016. Among the population, 425 accessions with sufficiently matured seeds harvested under the same conditions were selected for seed germination experiments.

\section{Phenotyping ABA sensitivity}

The harvested dry seeds were kept in a seed storage chamber with temperature at $20^{\circ} \mathrm{C}$ and relative humidity below $10 \%$. For germination evaluation, seeds of each accession were successively surface-sterilized with $70 \%$ ethanol for $2 \mathrm{~min}, 0.15 \% \mathrm{HgCl}_{2}$ solution for $15 \mathrm{~min}$, and then rinsed with sterile distilled water. Fifty sterilized seeds were transferred into plates $(9 \mathrm{~cm}$ petri dish) with half-strength Murashige and Skoog (1/2 MS) medium for the control check (CK) group or $1 / 2$ MS medium with $2 \mu \mathrm{M} \mathrm{ABA}$ for the treatment group. Three replicates were arranged for each group. All the abovementioned seeds in the medium were placed in a growth chamber $\left(26^{\circ} \mathrm{C}\right.$ and $100 \%$ relative humidity) under dark conditions. A seed was considered germinated when its radicle or coleoptile reached a length of $\geq 2 \mathrm{~mm}$. The percentage of germinated seeds in each plate was recorded on the 7th day after seeding. The mean values of three replicates of germination rates of CK (CKG) and ABA treatment group (ABAG) were used for association analysis. Relative germination rate (REG), calculated as (ABAG /CKG) $\times 100 \%$, was also used for association analysis because large variation in CKG exists in rice germplasms.

\section{Association mapping}

The genotypic data of the association population generated by next generation sequencing was collected from a previous study (Chen et al. 2014). The population structure was constructed by a random effect in linear mixed model (LMM) using the kinship (K) matrix, and GWAS was performed using LMM provided by the FaST-LMM programme (Lippert et al. 2011). The numbers of SNPs used for GWAS for the whole population, indica and japonica subpopulation were $3,916,415,2,767,159$ and $1,857,845$, respectively. All these SNPs in the whole population were used for principal component (PC) analysis by PLINK (Purcell et al. 2007). As some SNPs were closely linked, effective numbers of independent SNPs were determined by PLINK (window size 50, step size 50, $r^{2} \geq$ $0.2)$. Suggestive threshold was calculated based on the formula $-\log 10$ (1/effective number of independent SNPs) (Yang et al. 2014).

\section{Haplotype analysis}

The genomic DNA sequences of candidate genes were used for haplotype analysis among the 425 accessions (Chen et al. 2014). The trait differences between alleles of each SNP in the candidate genes were assessed by Student's $t$-tests. Among the significantly associated SNPs, the non-synonymous or other large effect SNPs/ InDels within gene coding regions and SNPs/InDels in promoter regions with relatively lower $P$ value were used for haplotype analysis.

\section{Expression level analysis}

Total RNA was isolated from rice leaves, which were collected from two-week-old rice seedlings treated by $100 \mu \mathrm{mol} \mathrm{ABA}$ for $0 \mathrm{~h}$ or $6 \mathrm{~h}$, by using TransZol reagent (TransGen Biotech, Beijing, China). RNA was reverse- 
transcribed by using EasyScript One-Step gDNA Removal and cDNA Synthesis SuperMix (TransGen Biotech, Beijing, China) according to the manufacturer's instructions. Relative quantitative PCR (qPCR) was performed in an optical 384-well plate with an Applied Biosystems ViiA7 real-time PCR system (Applied Biosystems, USA) by using SYBR Premix Ex-Taq reagent (TaKaRa, Tokyo, Japan). The qPCR was conducted with the rice ubiquitin gene as an internal control.

\section{Supplementary Information}

The online version contains supplementary material available at https://doi. org/10.1007/s44154-021-00011-4.

Additional file 1: Supplementary Table 1. Accession names and trait values (the mean of three replicates) for $C K G, A B A G$, and REG.

Additional file 2: Supplementary Table 2. Association loci of ABAG and REG in the indica subpopulation and whole population.

Additional file 3: Supplementary Table 3. Genes information in all association loci.

Additional file 4: Supplementary Table 4. Key SNPs and InDels in the three candidate genes.

Additional file 5: Supplementary Fig. 1. The relative expression levels of the three candidate genes under different stresses.

\section{Acknowledgements}

This work was supported by grants from the National Natural Science Foundation of China (31930080, 31821005).

\section{Authors' contributions}

LX conceived the experiments and supervised the project; LP conducted experiments with the assistance from $Z G, X L, S W$, and NW; TX analyzed the data with the assistance from YC, HT, and YY; TX and LX discussed the results and contributed to the final manuscript. The authors read and approved the final manuscript.

\section{Funding}

The funding has been properly acknowledged.

\section{Availability of data and materials}

All data and materials are available in the paper and online supplemental files.

\section{Declarations}

Ethics approval and consent to participate

Not relevant.

\section{Consent for publication}

All authors agree to publish.

\section{Competing interests}

Author LX is a member of the Editorial Board, but was not involved in the journal's review of, or decisions related to, this manuscript.

Received: 16 April 2021 Accepted: 8 September 2021

Published online: 07 October 2021

\section{References}

Allen GJ, Kuchitsu K, Chu SP, Murata Y, Schroeder JI (1999) Arabidopsis abi1-1 and abi2-1 phosphatase mutations reduce abscisic acid-induced cytoplasmic calcium rises in guard cells. Plant Cell 11(9):1785-1798. https://doi.org/10.11 05/tpc.11.9.1785

Batistič O, Rehers M, Akerman A, Schlücking K, Steinhorst L, Yalovsky S, Kudla J (2012) S-acylation-dependent association of the calcium sensor CBL2 with the vacuolar membrane is essential for proper abscisic acid responses. Cell Res 22(7):1155-1168. https://doi.org/10.1038/cr.2012.71

Bhandari A, Sandhu N, Bartholome J, Cao-Hamadoun TV, Ahmadi N, Kumari N, Kumar A (2020) Genome-wide qssociation study for yield and yield related traits under reproductive stage drought in a diverse indica-aus rice panel. Rice. 13(1):53. https://doi.org/10.1186/s12284-020-00406-3

Brocard-Gifford IM, Lynch TJ, Finkelstein RR (2003) Regulatory networks in seeds integrating developmental, abscisic acid, sugar, and light signaling. Plant Physiol 131(1):78-92. https://doi.org/10.1104/pp.011916

Chae MJ, Lee JS, Nam MH, Cho K, Hong JY, Yi SA, Suh SC, Yoon IS (2007) A rice dehydration-inducible SNF1-related protein kinase 2 phosphorylates an abscisic acid responsive element-binding factor and associates with ABA signaling. Plant Mol Biol 63(2):151-169. https://doi.org/10.1007/s11103-0069079-X

Chang Y, Nguyen BH, Xie Y, Xiao B, Tang N, Zhu W, Mou T, Xiong L (2017) Cooverexpression of the constitutively active form of OsbZIP46 and ABAactivated protein kinase SAPK6 improves drought and temperature stress resistance in rice. Front Plant Sci 8:1102. https://doi.org/10.3389/fpls.2017.011 02

Chen W, Gao Y, Xie W, Gong L, Lu K, Wang W, Li Y, Liu X, Zhang H, Dong H, Zhang W, Zhang L, Yu S, Wang G, Lian X, Luo J (2014) Genome-wide association analyses provide genetic and biochemical insights into natural variation in rice metabolism. Nat Genet 46(7):714-721. https://doi.org/10.103 8/ng.3007

Chen K, Li GJ, Bressan RA, Song CP, Zhu JK, Zhao Y (2020) Abscisic acid dynamics, signaling, and functions in plants. J Integr Plant Biol 62(1):25-54. https://doi.org/10.1111/jipb.12899

Cutler SR, Rodriquez PL, Finkelstein RR, Abrams SR (2010) Abscisic acid: emergence of a core signaling network. Annu Rev Plant Biol 61(1):651-679. https://doi.org/10.1146/annurev-arplant-042809-112122

Finkelstein RR, Lynch TJ (2000) The Arabidopsis abscisic acid response gene ABI5 encodes a basic leucine zipper transcription factor. Plant Cell 12(4):599-609. https://doi.org/10.1105/tpc.12.4.599

Finkelstein RR, Wang ML, Lynch TJ, Rao S, Goodman HM (1998) The Arabidopsis abscisic acid response locus ABI4 encodes an APETALA 2 domain protein Plant Cell 10(6):1043-1054. https://doi.org/10.1105/tpc.10.6.1043

Fujii H, Zhu JK (2009) Arabidopsis mutant deficient in 3 abscisic acid-activated protein kinases reveals critical roles in growth, reproduction, and stress. Proc Natl Acad Sci U S A 106(20):8380-8385. https://doi.org/10.1073/pnas. 0903144106

García-León M, Cuyas L, El-Moneim DA, Rodriguez L, Belda-Palazón B, SanchezQuant E, Fernández Y, Roux B, Zamarreño ÁM, García-Mina JM, Nussaume L, Rodriguez PL, Paz-Ares J, Leonhardt N, Rubio V (2019) Arabidopsis ALIX regulates stomatal aperture and turnover of abscisic acid receptors. Plant Cel 31(10):2411-2429. https://doi.org/10.1105/tpc.19.00399

Giraudat J, Hauge BM, Valon C, Smalle J, Parcy F, Goodman HM (1992) Isolation of the Arabidopsis ABI3 gene by positional cloning. Plant Cell 4(10):12511261. https://doi.org/10.1105/tpc.4.10.1251

Guo Z, Yang W, Chang Y, Ma X, Tu H, Xiong F, Jiang N, Feng H, Huang C, Yang $P$, Zhao H, Chen G, Liu H, Luo L, Hu H, Liu Q, Xiong L (2018) Genome-wide association studies of image traits reveal genetic architecture of drought resistance in Rice. Mol Plant 11(6):789-805. https://doi.org/10.1016/j.molp.201 8.03 .018

He Y, Hao Q, Li W, Yan C, Yan N, Yin P (2014) Identification and characterization of ABA receptors in Oryza sativa. PLoS One 9(4):e95246. https://doi.org/10.13 71/journal.pone.0095246

Huang X, Wei X, Sang T, Zhao Q, Feng Q, Zhao Y, Li C, Zhu C, Lu T, Zhang Z, Li M, Fan D, Guo Y, Wang A, Wang L, Deng L, Li W, Lu Y, Weng Q, Liu K, Huang T, Zhou T, Jing Y, Li W, Lin Z, Buckler ES, Qian Q, Zhang QF, Li J, Han B (2010) Genome-wide association studies of 14 agronomic traits in rice landraces. Nat Genet 42(11):961-967. https://doi.org/10.1038/ng.695

Huang X, Kurata N, Wei X, Wang ZX, Wang A, Zhao Q, Zhao Y, Liu K, Lu H, Li W, Guo Y, Lu Y, Zhou C, Fan D, Weng Q, Zhu C, Huang T, Zhang L, Wang Y, Feng L, Furuumi H, Kubo T, Miyabayashi T, Yuan X, Xu Q, Dong G, Zhan Q, Li C, Fujiyama A, Toyoda A, Lu T, Feng Q, Qian Q, Li J, Han B (2012) A map of rice genome variation reveals the origin of cultivated rice. Nature. 490(7421): 497-501. https://doi.org/10.1038/nature11532

Huijser C, Kortstee A, Pego J, Weisbeek P, Wisman E, Smeekens S (2000) The Arabidopsis SUCROSE UNCOUPLED-6 gene is identical to ABSCISIC ACID INSENSITIVE-4: involvement of abscisic acid in sugar responses. Plant J Cell Mol Biol. 23(5):577-585. https://doi.org/10.1046/j.1365-313x.2000.00822.x 
Irigoyen $M L$, Iniesto E, Rodriguez L, Puga MI, Yanagawa Y, Pick E, Strickland E, Paz-Ares J, Wei N, de Jaeger G, Rodriguez PL, Deng XW, Rubio V (2014) Targeted degradation of abscisic acid receptors is mediated by the ubiquitin ligase substrate adaptor DDA1 in Arabidopsis. Plant Cell 26(2):712-728. https://doi.org/10.1105/tpc.113.122234

Julian J, Coego A, Lozano-Juste J, Lechner E, Wu Q, Zhang X, Merilo E, BeldaPalazon B, Park SY, Cutler SR, An C, Genschik P, Rodriguez PL (2019) The MATH-BTB BPM3 and BPM5 subunits of Cullin3-RING E3 ubiquitin ligases target PP2CA and other clade A PP2Cs for degradation. Proc Natl Acad Sci U S A 116(31):15725-15734. https://doi.org/10.1073/pnas.1908677116

Kanwar P, Sanyal SK, Tokas I, Yadav AK, Pandey A, Kapoor S, Pandey GK (2014) Comprehensive structural, interaction and expression analysis of CBL and CIPK complement during abiotic stresses and development in rice. Cell Calcium 56(2):81-95. https://doi.org/10.1016/j.ceca.2014.05.003

Khew CY, Teo CJ, Chan WS, Wong HL, Namasivayam P, Ho CL (2015) Brassinosteroid insensitive 1-associated kinase 1 (OsI-BAK1) is associated with grain filling and leaf development in rice. J Plant Physiol 182:23-32. https:// doi.org/10.1016/j.jplph.2015.05.003

Kim H, Hwang H, Hong JW, Lee YN, Ahn IP, Yoon IS, Yoo SD, Lee S, Lee SC, Kim BG (2012) A rice orthologue of the ABA receptor, OsPYL/RCAR5, is a positive regulator of the $A B A$ signal transduction pathway in seed germination and early seedling growth. J Exp Bot 63(2):1013-1024. https://doi.org/10.1093/jxb/err338

Kim N, Moon SJ, Min MK, Choi EH, Kim JA, Koh EY, Yoon I, Byun MO, Yoo SD, Kim BG (2015) Functional characterization and reconstitution of ABA signaling components using transient gene expression in rice protoplasts. Front Plant Sci 6:614. https://doi.org/10.3389/fpls.2015.00614

Kobayashi Y, Yamamoto S, Minami H, Kagaya Y, Hattori T (2004) Differential activation of the rice sucrose nonfermenting1-related protein kinase2 family by hyperosmotic stress and abscisic acid. Plant Cell 16(5):1163-1177. https:// doi.org/10.1105/tpc.019943

Kobayashi Y, Murata M, Minami H, Yamamoto S, Kagaya Y, Hobo T, Yamamoto A, Hattori T (2005) Abscisic acid-activated SNRK2 protein kinases function in the gene-regulation pathway of ABA signal transduction by phosphorylating ABA response element-binding factors. Plant J Cell Mol Biol 44(6):939-949. https://doi.org/10.1111/j.1365-313X.2005.02583.X

Kong L, Cheng J, Zhu Y, Ding Y, Meng J, Chen Z, Xie Q, Guo Y, Li J, Yang S, Gong $Z$ (2015) Degradation of the ABA co-receptor ABI1 by PUB12/13 U-box E3 ligases. Nat Commun 6(1):8630. https://doi.org/10.1038/ncomms9630

Koornneef M, Bentsink L, Hilhorst H (2002) Seed dormancy and germination. Curr Opin Plant Biol 5(1):33-36. https://doi.org/10.1016/s1369-5266(01)00219-9

Leung J, Bouvier-Durand M, Morris PC, Guerrier D, Chefdor F, Giraudat J (1994) Arabidopsis ABA response gene ABI1: features of a calcium-modulated protein phosphatase. Science. 264(5164):1448-1452. https://doi.org/10.1126/ science.7910981

Leung J, Merlot S, Giraudat J (1997) The Arabidopsis ABSCISIC ACID-INSENSITIVE2 $(A B I 2)$ and $A B I 1$ genes encode homologous protein phosphatases $2 C$ involved in abscisic acid signal transduction. Plant Cell 9(5):759-771. https:// doi.org/10.1105/tpc.9.5.759

Li Z, Liu D (2012) ROPGEF1 and ROPGEF4 are functional regulators of ROP11 GTPase in ABA-mediated stomatal closure in Arabidopsis. FEBS Lett 586(9): 1253-1258. https://doi.org/10.1016/j.febslet.2012.03.040

Li X, Kong X, Huang Q, Zhang Q, Ge H, Zhang L, Li G, Peng L, Liu Z, Wang J, Li X, Yang Y (2019) CARK1 phosphorylates subfamily III members of ABA receptors. J Exp Bot 70(2):519-528. https://doi.org/10.1093/jxb/ery374

Lippert C, Listgarten J, Liu Y, Kadie CM, Davidson RI, Heckerman D (2011) FaST linear mixed models for genome-wide association studies. Nat Methods 8(10):833-835. https://doi.org/10.1038/nmeth.1681

Ma Y, Szostkiewicz I, Korte A, Moes D, Yang Y, Christmann A, Grill E (2009) Regulators of PP2C phosphatase activity function as abscisic acid sensors. Science. 324(5930):1064-1068. https://doi.org/10.1126/science.1172408

Magwa RA, Zhao H, Xing Y (2016) Genome-wide association mapping revealed a diverse genetic basis of seed dormancy across subpopulations in rice (Oryza sativa L.). BMC Genet 17:28. https://doi.org/10.1186/s12863-016-0340-2

Nakashima K, Yamaguchi-Shinozaki K (2013) ABA signaling in stress-response and seed development. Plant Cell Rep 32(7):959-970. https://doi.org/10.1007/s002 99-013-1418-1

Pandey GK, Grant JJ, Cheong YH, Kim BG, Li le G, Luan S (2008) Calcineurin-B-like protein CBL9 interacts with target kinase CIPK3 in the regulation of ABA response in seed germination. Mol Plant 1(2):238-248. https://doi.org/10.1 093/mp/ssn003
Park SY, Fung P, Nishimura N, Jensen DR, Fujii H, Zhao Y, Lumba S, Santiago J, Rodrigues A, Chow TFF, Alfred SE, Bonetta D, Finkelstein R, Provart NJ, Desveaux D, Rodriguez PL, McCourt P, Zhu JK, Schroeder Jl, Volkman BF, Cutler SR (2009) Abscisic acid inhibits type 2C protein phosphatases via the PYR/PYL family of START proteins. Science. 324(5930):1068-1071. https://doi. org/10.1126/science.1173041

Purcell S, Neale B, Todd-Brown K, Thomas L, Ferreira MA, Bender D, Maller J, Sklar P, de Bakker PI, Daly MJ, Sham PC (2007) PLINK: a tool set for whole-genome association and population-based linkage analyses. Am J Hum Genet 81(3): 559-575. https://doi.org/10.1086/519795

Rodriguez PL, Benning G, Grill E (1998) ABI2, a second protein phosphatase $2 C$ involved in abscisic acid signal transduction in Arabidopsis. FEBS Lett 421(3): 185-190. https://doi.org/10.1016/s0014-5793(97)01558-5

Saha J, Chatterjee C, Sengupta A, Gupta K, Gupta B (2014) Genome-wide analysis and evolutionary study of sucrose non-fermenting 1-related protein kinase 2 (SnRK2) gene family members in Arabidopsis and Oryza. Comput Biol Chem 49:59-70. https://doi.org/10.1016/j.compbiolchem.2013.09.005

Shang Y, Dai C, Lee MM, Kwak JM, Nam KH (2016) BRI1-associated receptor kinase 1 regulates guard cell ABA signaling mediated by open stomata 1 in arabidopsis. Mol Plant 9(3):447-460. https://doi.org/10.1016/j.molp.2015.12. 014

Shikha M, Kanika A, Rao AR, Mallikarjuna MG, Gupta HS, Nepolean T (2017) Genomic selection for drought tolerance using genome-wide SNPs in maize. Front Plant Sci 8:550. https://doi.org/10.3389/fpls.2017.00550

Soma F, Takahashi F, Yamaguchi-Shinozaki K, Shinozaki K (2021) Cellular Phosphorylation Signaling and Gene Expression in Drought Stress Responses: ABA-Dependent and ABA-Independent Regulatory Systems. Plants 10(4). https://doi.org/10.3390/plants10040756

Takahashi Y, Zhang J, Hsu PK, Ceciliato PHO, Zhang L, Dubeaux G, Munemasa S, Ge C, Zhao Y, Hauser F, Schroeder II (2020) MAP3Kinase-dependent SnRK2kinase activation is required for abscisic acid signal transduction and rapid osmotic stress response. Nat Commun 11(1):12. https://doi.org/10.1038/s414 67-019-13875-y

Tang N, Zhang H, Li X, Xiao J, Xiong L (2012) Constitutive activation of transcription factor OsbZIP46 improves drought tolerance in rice. Plant Physiol 158(4):1755-1768. https://doi.org/10.1104/pp.111.190389

Tischer SV, Wunschel C, Papacek M, Kleigrewe K, Hofmann T, Christmann A, Grill E (2017) Combinatorial interaction network of abscisic acid receptors and coreceptors from Arabidopsis thaliana. Proc Natl Acad Sci U S A 114(38): 10280-10285. https://doi.org/10.1073/pnas.1706593114

Umezawa T, Sugiyama N, Mizoguchi M, Hayashi S, Myouga F, YamaguchiShinozaki K, Ishihama Y, Hirayama T, Shinozaki K (2009) Type 2C protein phosphatases directly regulate abscisic acid-activated protein kinases in Arabidopsis. Proc Natl Acad Sci U S A 106(41):17588-17593. https://doi.org/1 0.1073/pnas.0907095106

Vlad F, Rubio S, Rodrigues A, Sirichandra C, Belin C, Robert N, Leung J, Rodriguez PL, Laurière C, Merlot S (2009) Protein phosphatases $2 C$ regulate the activation of the Snf1-related kinase OST1 by abscisic acid in Arabidopsis. Plant Cell 21(10):3170-3184. https://doi.org/10.1105/tpc.109.069179

Wang P, Zhao Y, Li Z, Hsu CC, Liu X, Fu L, Hou YJ, du Y, Xie S, Zhang C, Gao J, Cao M, Huang X, Zhu Y, Tang K, Wang X, Tao WA, Xiong Y, Zhu JK (2018a) Reciprocal regulation of the TOR kinase and ABA receptor balances plant growth and stress response. Mol Cell 69(1):100-112 e6. https://doi.org/10.101 6/j.molcel.2017.12.002

Wang K, He J, Zhao Y, Wu T, Zhou X, Ding Y, Kong L, Wang X, Wang Y, Li J, Song CP, Wang B, Yang S, Zhu JK, Gong Z (2018b) EAR1 negatively regulates ABA signaling by enhancing 2 C protein phosphatase activity. Plant Cell 30(4):815834. https://doi.org/10.1105/tpc.17.00875

Wu Q, Zhang X, Peirats-Llobet M, Belda-Palazon B, Wang X, Cui S, Yu X, Rodriguez PL, An C (2016) Ubiquitin ligases RGLG1 and RGLG5 regulate abscisic acid signaling by controlling the turnover of phosphatase PP2CA. Plant Cell 28(9):2178-2196. https://doi.org/10.1105/tpc.16.00364

Xiang Y, Sun X, Gao S, Qin F, Dai M (2017) Deletion of an endoplasmic reticulum stress response element in a ZmPP2C-A gene facilitates drought tolerance of maize seedlings. Mol Plant 10(3):456-469. https://doi.org/10.1016/j.molp.201 6.10 .003

Yu X, Niu X, Yang S, Li Y, Liu L, Tang W, Liu Y (2011) Research on heat and drought tolerance in rice(Oryza sativa L.) by overexpressing transcription factor OsbZIP60. Scientia Agricultura Sinica 44(20):4142-4149. https://doi. org/10.3864/j.issn.0578-1752.2011.20.002 
Yang W, Guo Z, Huang C, Duan L, Chen G, Jiang N, Fang W, Feng H, Xie W, Lian X, Wang G, Luo Q, Zhang Q, Liu Q, Xiong L (2014) Combining highthroughput phenotyping and genome-wide association studies to reveal natural genetic variation in rice. Nat Commun 5(1):5087. https://doi.org/10.1 038/ncomms6087

Yu F, Qian L, Nibau C, Duan Q, Kita D, Levasseur K, Li X, Lu C, Li H, Hou C, Li L, Buchanan BB, Chen L, Cheung AY, Li D, Luan S (2012) FERONIA receptor kinase pathway suppresses abscisic acid signaling in Arabidopsis by activating ABI2 phosphatase. Proc Natl Acad Sci U S A 109(36):14693-14698. https://doi.org/10.1073/pnas.1212547109

Yuan L, Xie GZ, Zhang S, Li B, Wang X, Li Y, Liu T, Xu X (2021) GmLCLs negatively regulate $A B A$ perception and signalling genes in soybean leaf dehydration response. Plant Cell Environ 44(2):412-424. https://doi.org/10.1111/pce.13931

Zhang L, Li X, Li D, Sun Y, Li Y, Luo Q, Liu Z, Wang J, Li X, Zhang H, Lou Z, Yang $Y$ (2018) CARK1 mediates ABA signaling by phosphorylation of ABA receptors. Cell Discov 4(1):30. https://doi.org/10.1038/s41421-018-0029-y

Zhao Z, Xu J, Chen J, Kim S, Reimers M, Bacanu SA, Yu H, Liu C, Sun J, Wang Q, Jia P, Xu F, Zhang Y, Kendler KS, Peng Z, Chen X (2015) Transcriptome sequencing and genome-wide association analyses reveal lysosomal function and actin cytoskeleton remodeling in schizophrenia and bipolar disorder. Mol Psychiatry 20(5):563-572. https://doi.org/10.1038/mp.2014.82

Zhou X, Hao H, Zhang Y, Bai Y, Chang GX (2015) PKS5/CIPK11, a SnRK3-type protein kinase, is important for ABA responses in arabidopsis through phosphorylation of ABI5. Plant Physiol 168(2):659-676. https://doi.org/10.11 04/pp.114.255455

Zong W, Tang N, Yang J, Peng L, Ma S, Xu Y, Li G, Xiong L (2016) Feedback regulation of ABA signaling and biosynthesis by a bZIP transcription factor targets drought-resistance-related genes. Plant Physiol 171(4):2810-2825. https://doi.org/10.1104/pp.16.00469

\section{Publisher's Note}

Springer Nature remains neutral with regard to jurisdictional claims in published maps and institutional affiliations. 